\title{
The Stratifying Role of Job level for Sickness Absence and the Moderating Role of Gender
}

\section{and Occupational Gender Composition}

\author{
by KRÖGER, Hannes
}

\section{Abstract}

The study investigates whether sickness absence is stratified by job level - understood as the authority and autonomy a worker holds - beyond the association with education, income, and occupation. A second objective is to establish the moderating role of gender and occupational gender composition on this stratification of sickness absence. Four competing hypotheses are developed that predict different patterns of moderation. Associations between job level and sickness absence are estimated for men and women in three groups of differing occupational gender composition, using data from the German Socio-economic Panel Study (SOEP). For the purpose of moderation analysis, this study employs a new method based on Bayesian statistics, which enables the testing of complex moderation hypotheses. The data support the hypothesis that the stratification of sickness absence by job level is strongest for occupational minorities, meaning men in female-dominated and women in male-dominated occupations.

Keywords: Occupational sex segregation, Career mobility, Promotions, Gender,

Absenteeism, Bayesian inference 


\section{$1 \quad 1$ Introduction}

The persistence of health inequalities despite the health care provision of modern welfare states is a well-established finding (Mackenbach, 2012). One point of discussion is the importance of the labor market in creating not only social inequalities, but also health inequalities (Hannes Kröger, Pakpahan, \& Hoffmann, 2015; Mackenbach \& Bakker, 2003). One particular aspect that closely links labor market-related inequalities and health inequalities is sickness absence, which reflects both the health of the individual worker, but also their labor market-related health behavior (Marmot, Feeney, Shipley, North, \& Syme, 1995; North et al., 1993). While other dimensions of health inequality are often related to education, occupation, and income, sickness absence has been attributed more than other aspects of health to the job level within an occupation (Beemsterboer, Stewart, Groothoff, \& Nijhuis, 2009), with job level understood as the degree of an employee's autonomy and authority in everyday work, regardless of their occupation.

A crucial aspect in the relationship between job level and sickness absence is the role of gender, because there are considerable differences between men and women both with regard to job level and sickness absence. Women have a higher degree of sickness absence and typically occupy lower status jobs (Alexanderson, Leijon, Åkerlind, Rydh, \& Bjurulf, 1994; Baxter \& Wright, 2000). We therefore surmise that the stratifying mechanisms linking job level to sickness absence are different for men and women. However, not only gender, but also occupational gender composition (Fried, Melamed, \& Ben-David, 2002; Mastekaasa \& Melsom, 2014) has a considerable influence on the behavior of employees, and we will show it is actually a combination of these two factors that best explains differences in the stratification of sickness absence. 
This study makes three important contributions to the social science literature. First, the stratification of sickness absence by job level is estimated for workers on the German labor market. Second, four competing hypotheses regarding the moderating effect of gender and occupational gender composition on the relationship between job level and sickness absence are theoretically developed and empirically tested. Third, we introduce a new Bayesian method (Hoijtink, Klugkist, \& Boelen, 2008; van de Schoot, Verhoeven, \& Hoijtink, 2013), developed in psychometric literature, which is well suited for testing complex moderation hypotheses.

\subsection{Previous research on sickness absence and job status}

Many studies have examined the gender gap in sickness absence between men and women. It is usually agreed that men have fewer days of sickness absence, but there is little agreement on the explanations for this gender gap (Bekker, Rutte, \& van Rijswijk, 2009). Another approach to the relationship between gender and sickness absence is to focus on occupational gender segregation. Most studies find some support for a U-shaped correlation of gender composition and sickness absence, meaning that lowest levels of sickness absence are found in mixed occupations (Alexanderson et al., 1994; Bekker et al., 2009; Evans \& Steptoe, 2002; Knutsson \& Goine, 1998; Leijon, Hensing, \& Alexanderson, 2004).

Another strand of research consistently confirms that different measures of job level such as job control, autonomy, skill-level, wages, or job security are strongly related to the incidence and duration of sickness absence. The higher the level or the better the conditions for the employee are, the lower the rate of sickness absence is (Beemsterboer et al., 2009; Johns, 2010; Pines, Skulkeo, Pollak, Peritz, \& Steif, 1985; Sharp \& Watt, 1995; Vahtera, Kivimäki, 

However, there has been no systematic investigation of the moderating effect of gender and gender composition on the relationship between sickness absence and job status, which is the purpose of this study.

\section{$6 \quad 2$ Theoretical framework}

\section{$7 \quad 2.1$ Explanations for the association between job level and sickness absence}

We define job level as the level of autonomy - achieved through expertise and skill - and authority within the production process that is attributed to the position that an individual worker occupies. The rationale behind the expectation of superior outcomes in sickness absence (lower rates of absence) for higher levels of job level is based on three general mechanisms. First, higher job levels correspond to stronger pressure for workers to monitor themselves to be more productive. Employees' feelings of goals shared with their employer or the company itself are much higher than at lower levels of authority and autonomy (Wright \& Perrone, 1977). Furthermore, high pressure, workload, and responsibilities for workers in high status positions can encourage presenteeism, which is the tendency to show up at work despite an acute illness. As these pressures and demands are accompanied by considerable rewards, be they financial or otherwise, the balance between job-related efforts and rewards (Siegrist, 1996, 2009) is maintained and commitment strengthened. Higher rewards counteract job strain (Karasek, 1979), which is more prevalent at lower job levels and might induce absenteeism (Kuper \& Marmot, 2003). 
The second mechanism that drives the systematic association between job level and sickness absence is selection of employees for advancement to higher job levels in a company. Employers prefer to give positions of elevated authority and autonomy to those employees who identify more strongly with the company and do not see themselves in a structural opposition of interests to their employer. Therefore, the degree of sickness absence is not only a more easily observable indicator of productivity due to the actual time that is missed at work, but also a proxy for motivation and commitment (Mastekaasa, 1996). This mechanism will also increase stratification of sickness absence according to job level, as it selects those with already low levels of absence to higher job levels.

Third, workers at higher job levels might be healthier in general and therefore simply need to take fewer days off than workers in lower status jobs. We control for this mechanism in our empirical analysis, as it is not specific to sickness absence, but represents general patterns of health inequalities. The interesting aspect in distinguishing between the first two mechanisms is to investigate how they might be moderated by gender and occupational gender composition, which allows for predictions about the degree of stratification in different groups on the labor market to be made. The literature has mostly treated occupational gender composition separately from individual gender, but we argue that a joint view helps in understanding complex patterns of job status stratification.

\subsection{Four hypotheses about the moderating effect of gender and occupational gender}

\section{composition}

For the purpose at hand, we identify four larger strands of theoretical literature that help guide specific hypotheses about the moderating effect of gender composition on the stratification of sickness absence by job level. 
First, gender roles lead to different expectations about the focus that men and women should have in life (Eagly, 1987). Traditionally, men are expected to be wage earners, while women are traditionally expected to care for home and family, but often have to face the double burden of both paid employment and unpaid domestic work. As a self-regulatory mechanism, men with higher job levels are likely to allow themselves less absence than both women at similar job levels and men with lower job levels, based on gendered role expectations.(Eagly, Wood, \& Diekman, 2000, p. 149).

Second, we draw on the idea of different masculinities to hypothesize that, in maledominated occupations, a male culture of presenteeism will become part of a hegemonic masculinity (Raewyn W Connell, 1987, pp. 184-188) to which women also adapt in order to further their career (R. W. Connell \& Messerschmidt, 2005, p. 847).

Third, we turn to theories that posit that numerical minority status can be the defining characteristic for interactions within groups (Blau, 1977; Kanter, 1977a). This theoretical view is symmetric, expecting the same consequences for men in female-dominated as for women in male-dominated settings. It proposes visibility, polarization, and assimilation as the three core mechanisms that distinguish how the minority is treated in contrast to the majority (Kanter, 1977b).

Fourth, minority status can be thought of not as a numerical concept limited to workplace, but in terms of subordinate groups within the larger societal structure (Blalock, 1967), which would here apply to women in the labor market context. Conversely, men in femaledominated occupations might not only be free of the negative consequences of their token status, but could actually benefit from their structurally superior status. 
We develop four hypotheses $\left(\boldsymbol{H}_{1 \boldsymbol{a}}-\boldsymbol{H}_{1 \boldsymbol{d}}\right)$ for the moderating effect of gender and gender composition on the relationship between sickness absence and job level, based on these theoretical observations.

The gender role hypothesis refers to the individual's gender, and predicts that the stratifying effect of job level will be stronger for men than for women $\left(\boldsymbol{H}_{\mathbf{1} a}\right)$. The hypothesis is rooted in differences in gender roles. A great deal of research has shown that women put more emphasis on maintaining a healthy lifestyle and treating illnesses or mental problems than men do (Cockerham, 2005; Dean, 1989), while men more often have a dismissive attitude towards health risks (Peate, 2004). As a result, men are more likely than women to go to work even when they are ill, which also conforms with gendered role expectations of men as the primary wage earners. This difference will manifest itself particularly clearly in higher status positions, where absence is generally lower and job demands are high (Demerouti, Blanc, Bakker, Schaufeli, \& Hox, 2009; Kuoppala, Lamminpää, Liira, \& Vainio, 2008). Furthermore, employed women often face a double burden in which they have to take care of the household and children or are much more often primary care takers of the elderly than men are (Bratberg, Dahl, \& Risa, 2002; Doress-Worters, 1994; Mastekaasa, 2000). Compared to men, women have an increased risk of having to take sick leave not for themselves but to care for their children (Floderus, Hagman, Aronsson, Marklund, \& Wikman, 2012) or other family members to resolve the work-family conflict (Clays, Kittel, Godin, Bacquer, \& Backer, 2009; Jansen et al., 2006) and comply with role expectations as main caregiver, instead of the expectations generated by job responsibility. This prediction should hold for women in any kind of occupational group defined by gender composition. 
The male culture of presenteeism hypothesis refers to the occupational context and proposes that, irrespective of individual gender, the stratification of sickness absence by job level should be stronger in male-dominated than in mixed and female-dominated occupations $\left(\mathbf{H}_{\mathbf{1 b}}\right)$. A male culture of presenteeism (Simpson, 1998) is defined as a common set of attitudes and behavior within an occupation that determines employees' absence behavior, due to strong competitiveness which may be directly or indirectly reinforced by the expectations of employers and supervisors (Nicholson \& Johns, 1985, p. 400). In maledominated occupations, short periods of recovery and a more dismissive attitude towards illness in general (Cockerham, 2005; Dean, 1989) can become the norm for all workers, regardless of individual gender or individual attitudes. Low absenteeism can become part of the image of the ideal worker (Acker, 1990; J. Williams, 2001), which is often an image dominated by masculinity (Turco, 2010). This can lead to a spillover effect, with individual male behavior defining how a worker should behave in a male-dominated occupation (Pierce, 1996). This leads to a shared cultural ideal favoring presenteeism within maledominated occupations, reflecting a hegemonic masculinity (Raewyn W Connell, 1987, pp. 184-188) to which women also adapt to further their career (R. W. Connell \& Messerschmidt, 2005, p. 847). Consequently, employees in male-dominated occupations will be less likely to call in sick due to the pressure of being present, especially in higher status positions with more responsibility. Equally, employers in male-dominated occupations will notice sickness absence more often, will expect higher presence rates from their employees, and will favor those for promotion who are less often absent from work (Demerouti et al., 2009; Simpson, 1998; Watts, 2009). 
The occupational minority hypothesis is based on theories about token or occupational minority status (Kanter, 1977a; Taylor, 2010). It predicts that persons in an occupational minority position will reduce their sickness absence to a higher degree if they occupy high status positions. In addition, sickness absence will have a stronger negative effect on future job level for occupational minorities. Combined, both mechanisms are expected to be stronger for men in female-dominated occupations and for women in male-dominated occupations $\left(\boldsymbol{H}_{1 c}\right)$. Both men and women are influenced by common elements of their occupation in their daily roles, whether they work in a segregated workplace or not (Taylor, 2010). In contrast to the male culture of presenteeism hypothesis, the occupational minority hypothesis attributes the dominance not to hegemonic masculinity in male-dominated occupations, but to the numerical majority position per se. This implies negative consequences for promotion chances and rewards in general for an individual in a minority position, regardless of gender. Consequently, to make up for the disadvantages experienced due to homophily and status expectations, persons with minority status need to outperform the majority group (Kanter, 1977a). Their sickness absence will be noted more often and will consequently have a stronger impact on their chances of promotion. In addition, individuals in a minority position will try to (over) compensate for their minority status and the resulting attention and scrutiny by further reducing their absence when they have reached a position of higher status and responsibility. Occupational minorities will thus experience a stronger stratification between sickness absence and job level than occupational majorities.

Finally, the so-called glass escalator hypothesis (Hultin, 2003; C. L. Williams, 1992, 2013) claims that men in female-dominated occupations have a general advantage in terms of career prospects, despite being a numerical minority. Therefore, men in female-dominated 
occupations are selected less on their previous levels of sickness absence when considered for positions of higher authority than women in female-dominated occupations. It follows that the relationship between sickness absence and job level should be weaker for men than for women in female-dominated occupations $\left(\boldsymbol{H}_{\mathbf{1} \boldsymbol{d}}\right)$.

The original glass escalator hypothesis proposed by Williams (C. L. Williams, 1992) states that coworkers welcome men in female-dominated occupations as bearers of potential prestige and pay rises, giving them a better standing within the occupational field, which gives weight to the argument that men occupy a structurally higher power position (Blalock, 1967) despite being in numerical minority. At the same time, clients who are used to women in female-dominated jobs expect to deal with women rather than men; clients' expectations are very important in many female-dominated occupations, because in practice these occupations involve high volume interaction with clients on a day-to-day basis (e.g. care jobs, nursing, social work). Thus, to circumvent client disapproval, men are promoted to higher or supervisory positions which require less client interaction (Hultin, 2003). Transferred to the problem at hand, the glass escalator hypothesis proposes that sickness absence should be less important for future job level for men than for women in female-dominated occupations, because men face weaker competition for promotion. Furthermore, men need to expend less effort than women, relatively speaking, once they have reached higher positions in female-dominated jobs. Following the last hypothesis, the general mechanisms linking sickness absence and job level are overridden by the glass escalator. Consequently, if overall selectiveness for men is reduced, selection for sickness absence should also be reduced for men. This leads to the expectation that male job level should be less related to sickness absence than is the case for women. 
Germany provides an interesting example for the analysis of sickness absence and job level, because short-term sickness absence is not associated with a loss of income for employees. Continuation of wage payments by the employer for short-term absences (less than six weeks) is mandatory by law. This results in a $100 \%$ wage replacement rate during sick leave, which makes the regulation of sickness absence in Germany one of the most generous in the world (Scheil-Adlung \& Sandner, 2010). At the same time, employment protection is strict enough that smaller incidences of sickness absence are usually not a sufficient reason for a decision to call in sick is less dictated by short-term financial considerations or by worries about immediate dismissal. Rather, it can be seen as a medium or long-term strategy of balancing the health advantages and job disadvantages of sickness-related absence. Despite the generosity of the sick leave regulation, the average degree of sickness absence in 
2

\subsection{Data}

We test the four hypotheses using 5 waves of panel data from the German Socio-Economic Panel Study (SOEP), version 31.1 ((SOEP) Socio-Economic Panel, 2015). The analysis of the relationship between job level and sickness absence is conducted separately for men and women in three categories of occupational gender composition, yielding six fundamental groups to be investigated. The groups are men and women each in male-dominated, mixed, and female-dominated occupations. The construction of the groups is discussed in detail below. The SOEP is a household survey with annual interviews, and has been conducted since 1984. The survey currently includes more than 20,000 individuals in more than 10,000 households. The households are contacted through a stratified random sampling procedure and are representative of the German household population. Interviews are conducted face-to-face by trained interviewers (Wagner, Frick, \& Schupp, 2007). For the analyses, we make use of 5 waves $(2002,2004,2006,2008,2010)$. Only those waves which contain data on mental and physical health are retained. The SOEP consists of its original sample from 1984 and several refreshment samples.

\subsection{Sample}

The sample is restricted in the following way: We exclude the self-employed, persons in vocational or educational training, all employees in the public sector, all those younger than 18 or older than 64 , and all those who are not currently employed. We focus on employees from the private sector because, in the public sector, rules regulating promotions are more bureaucratic and job security is much stronger. Therefore, the link between sickness 
absence and job level will not follow the theory outlined in this paper and cannot be compared to the private sector. Lastly, all observations with missing values on sickness absence or any of the predictors are excluded, as other forms of treatment of missing values are currently not supported by the method implemented in this study. The restrictions result in a sample of 16,384 individuals and 87,573 observations. Table $C$ in the online supplement compares the sample statistics of the observations which are included in the study to the ones excluded due to missing values on covariates. The table shows that missing observations have higher days of sickness absence and more often have low status jobs, lower wages, and lower EGP categories. While the differences are not dramatic, this indicates that the results reported here might be an underestimation of the stratification of sickness absence by job level.

\subsection{Measurements}

The dependent variable in the analyses is sickness absence. We use the number of selfreported days of sickness absence in the current year. Reported days of sickness absence have been shown to be strongly associated to records of actual sickness absence from health insurance register data (Ferrie et al., 2005).

An individual's job level is based on categorizations of their position within their company's hierarchy, corresponding to the degree of autonomy, skill, and authority (Hoffmeyer-Zlotnik, 2003; H Kröger, 2016). It is not linked to occupational classifications, but measured in a separate question in the survey. The resulting scale is categorized as presented in table A of the online supplement. The lowest group consists of unskilled or semi-skilled workers. The second group consists of skilled blue-collar workers and white-collar workers with basic tasks. The third group comprises white-collar workers with qualified tasks and 
responsibilities, as well as (assistant) foremen among the blue-collar workers. The fourth group includes highly qualified white-collar workers with some degree of supervisory duties as well as master craftsmen (German: Meister) and senior foremen (German: Polier). The highest group consists of white-collar middle and senior management with extensive supervisory duties. We collapse the highest two categories (4 and 5), because the highest category (managers and supervisors) does not have enough observations in femaledominated occupations for both men and women (20 and 16 observations respectively). The reference in the analyses is always the lowest category of job level.

More common measurements associated with job or labor market status like the EGP classification (Erikson, Goldthorpe, Portocarero, \& Lucienne, 1979) or ISEI (Ganzeboom, De Graaf, \& Treiman, 1992) cannot be used for our research question, because they are designed to reflect also differences between occupations, while the focus of this study is strictly on the hierarchy within occupations and companies. Furthermore, the job level measurement does not distinguish between blue and white collar workers, as each of the final four categories contains both blue and white collar workers. Additionally, occupational class based on the EGP classifications will function as a control variable in the model, to ensure that differences in job level do not merely reflect differences between occupations. This approach to measuring job level also differs from measurements of job decision latitude in job strain models (Karasek, 1979) or reward dimensions in the effort-rewardimbalance model (Siegrist, 1996). Both approaches focus on occupational autonomy but while they directly measure this autonomy on a scale, job level as used here is the formal level ar the workplace that is a predictor of actual job autonomy. 
We apply the same division of occupational groups in this study as Hultin (Hultin, 2003), who divides occupations into three types: Those with fewer than $30 \%$ women are defined as male-dominated; those occupations with more than $70 \%$ women are called femaledominated occupations, and all occupations in between are referred to as mixed occupations. We use data from the German Federal Statistical Office (FSO) on gender proportions in occupational groups, using their Klassifizierung der Berufe 92 (KLDB-92) (similar to ISCO-88) classification scheme on the 3-digit level. Figure A in the online supplement shows the proportion of females (averaged over the period of observation) in the five occupations with the highest prevalence in the sample for each occupational gender segregation category. The occupations are labelled according to the ISCO-88 equivalent of the KLDB-92 classification. Male-dominated occupations include haulage, mechanics, and engineering, while female-dominated occupations include cleaning, nursing, and sales, reflecting a relatively traditional gender division of occupations in Germany (see also Busch, 2013, Chapter 7).

The estimation of stratification of sickness absence by job level is made conditional on three common dimensions of social stratification and current health status. Education is categorized into primary, secondary, and tertiary education according to the CASMIN classifications (König, Lüttinger, \& Müller, 1988). Labor income is measured as log. monthly gross wage. Finally, occupational class is classified according to the Erikson-GoldthorpePortocarero typology (Erikson et al., 1979). Additionally, age is taken into account, as it is a natural confounder for the association of sickness absence and job level, since it is positively related to both variables. Current health status is measured with standardized scores from the physical and mental component of the SF-12 item battery from the SOEP (Andersen, Mühlbacher, Nübling, Schupp, \& Wagner, 2007). Table B lists the corresponding variables in 
1 detail and table $\mathrm{C}$ presents a summary of statistics for all variables used in the analyses (see

2 online supplementary). All data management and descriptive statistics were conducted

3 using Stata 14.1 and additional user written software (Jann, 2007). 
We estimate a zero-inflated negative binomial model with a random intercept for individuals. This model is appropriate for the distribution of the number of days of sickness absence, which is a count variable, for which the mean is unequal to the variance and has excess numbers of zero. The parameters are relative rates, meaning they reflect relative change rather than change in absolute numbers of days of sickness absence. We also replicate the results using linear regression, where the absolute differences in number of $9 \quad$ sickness days are estimated and compared.

The models include a random intercept for each individual, capturing time constant characteristics. The estimated coefficients are weighted averages of the association within and between individuals, weighted by the relative proportion of variance within and between individuals. This weighting reflects the fact that social stratification occurs both between individuals and within an individual's life-course (which could also be called intragenerational mobility).

Formally, the negative binomial model is defined as:

$$
\log \left(S A_{i t}\right)=\alpha_{S A}+\sum_{t}^{4} \beta_{r} D_{J S_{\text {rit }}}+\boldsymbol{\gamma} \boldsymbol{Z}_{i t}+u_{i}(1)
$$

The linear model is defined as:

$$
S A_{i t}=\alpha_{S A}+\sum_{-2}^{4} \beta_{r} D_{J S_{r i t}}+\boldsymbol{\gamma} \boldsymbol{Z}_{i t}+u_{i}+e_{i t}(2)
$$


$7 \quad D_{J S_{\text {rit }}}=1$ if $J S_{i t}=r$ and sickness absence by job level.

The individuals are indexed by $i$, the year by $\mathrm{t}$. The $\beta$ s are the coefficients of interest; $\gamma$ is a vector of coefficients of the association of sickness absence with education, wages, occupational class, health and age. The parameters of the equation are estimated for the six main groups under study, constituted by a cross-tabulation of gender and occupational gender composition. The $D_{J S_{r}}$ stands for the dummy variables of job level which take the value 1 and 0 under the conditions: $D_{J S_{\text {rit }}}=0$ if $J S_{i t} \neq r ; r=\{$ low, middle, high $\}$.

We use the lowest status category as a reference category. Based on this model specification, we will evaluate the four hypotheses (table 1) developed in the theory section. If a comma separates coefficients it means no ordering is assumed. Otherwise, a greater or lesser sign indicates which ordering of the coefficients the theory expects. The alternative hypothesis $\boldsymbol{H}_{2}$ states that there is no systematic ordering of the degree of stratification of 


\section{$2 \quad 3.5$ Bayesian evaluation of inequality constrained hypotheses}

One of the key features of this study is its application of a new method, developed in psychometric research (Hoijtink et al., 2008; van de Schoot et al., 2013), to an investigation of the moderating effect of gender and occupational gender composition. This approach allows us to determine which of the hypotheses $\left(\boldsymbol{H}_{1 \boldsymbol{a}}-\boldsymbol{H}_{2}\right)$ receives most support from the data and modeling strategy in terms of relative probability (e.g. with $80 \%$ probability $\boldsymbol{H}_{\mathbf{1} \boldsymbol{a}}$ is true compared to the other hypotheses). As the hypotheses are complex in their predictions, it is helpful to apply an evaluation strategy that yields straightforward interpretations of degrees of support for the hypotheses.

As this method for evaluating hypotheses is new in social sciences, the online supplement (S2) gives a brief introduction, using as an example one of the hypotheses from this study. The general modeling approach we use is called Integrated Nested Laplace Approximation (INLA) (Rue, Martino, \& Chopin, 2009), implemented as a package for R (www.r-inla.org).

\section{Results}

Section S3 in the online supplement gives descriptive evidence on the relationship between sickness absence, job level, and occupational gender composition.

The estimates from the Bayesian model are presented in figures 1 and 2 (full model results are documented in $\$ 4$ in the online supplement). The dots in the figures plot the mean of the posterior distribution of the coefficients from the regression of sickness absence onjob level for the six groups under investigation. The lines represent the $95 \%$ credibility interval. 
In figure 1 the coefficients are reported as incidence rate ratios (IRR).

In male-dominated occupations, women with higher job levels have $51 \%$ lower rates of sickness absence than women from the lowest job levels in this group. For women in mixed and female-dominated occupations the gradient is smaller, with incidences of sickness absence reduced by $17 \%$ and $29 \%$ respectively when comparing the highest job level to the lowest.

Sickness absence is also stratified for men in all occupational groups. The degree of stratification is lowest (no or an inverse gradient) in mixed occupations, stronger in maledominated occupations (highest job level: 13\% reduced incidence), and strongest in femaledominated occupations, with a reduction of $49 \%$ between the highest job level and the lowest. In general, we can see that each step up in job level decreases sickness absence in all six groups, except for men in mixed occupations. However, the step to the highest job level is consistently the largest; other steps (e.g. between lower and lowest level) are smaller, often with less than $10 \%$ difference, which we regard as a substantially small effect. When we ran the same models using a linear regression model, we found a very similar pattern. Figure 2 shows the coefficients from this model. The coefficients can now be interpreted as the number of days of sickness absence. The absolute differences show even clearer hierarchical stratification within each group, and the overall pattern between the groups is also similar. Most coefficients range between 2 and 8 days of sickness absence, showing the substantial size of the stratification in absolute terms, in addition to the relevant relative differences presented in figure 1 . regarding the moderating effects of gender and occupational gender composition. The first 
column lists the four hypotheses under scrutiny, plus the alternative hypothesis of no ordering $\left(\boldsymbol{H}_{\mathbf{1} \boldsymbol{a}}-\boldsymbol{H}_{\mathbf{1} d}, \boldsymbol{H}_{\mathbf{2}}\right)$. The second column reports the prior expectation regarding the probability that the respective hypothesis will receive support, assuming that there is no systematic ordering of the coefficients. The third column shows the observed support from the posterior distribution. The ratio of those two quantities is the Bayes Factor (BF), which is shown in column four and signifies the degree of support for the respective hypothesis. The last column contains the posterior model probabilities (PMP), which can be interpreted as the relative support of one hypothesis compared to all other hypotheses.

\section{From the comparison of the BF we see immediately that the occupational minority} hypothesis is extremely well-supported, while all other hypotheses receive only little (BF below 1) support from the data and the model. The BF for hypothesis $\boldsymbol{H}_{\mathbf{1} \boldsymbol{c}}$ indicates that the hypothesis in question receives about 223 times more support than the hypothesis of no (or random) ordering. This represents very strong support for the hypothesis that the association of job level with sickness absence is stronger for numerical minorities - men in female-dominated and women in male-dominated occupations - than for men and women in all other occupational groups. The reason for this high Bayes Factor is the high specificity in the predictions of $\boldsymbol{H}_{\mathbf{1}}$. It orders three sets of six coefficients, yet there is still considerable agreement in the posterior distribution (6.6\%) with this prediction. This is mirrored in the predictive model probability, which shows that among the set of hypotheses tested in this study, hypothesis $\boldsymbol{H}_{\mathbf{1}}$ is likely to be correct with a probability of approximately $99 \%$. The evaluation of the linear regression model yields the same relative support. The occupational minority hypothesis receives even more support in the absolute difference specification than in the relative model specification (BF 339). 


\section{Discussion}

In this study, we investigated the relationship between sickness absence and job level. A special focus was put on the moderating effects of gender and occupational gender composition. We introduced four hypotheses that proposed different moderating patterns of gender and occupational gender composition on the stratifying effect of job level.

The analyses primarily supported the occupational minority hypothesis $\left(\boldsymbol{H}_{\mathbf{1}}\right)$. Men in female-dominated and women in male-dominated occupations show stronger stratification of sickness absence by job level. It seems that higher job levels are more consequential for the numerical minority on the labor market when it comes to sickness absence, as proposed in the broader theories of Kanter (1977a) and Taylor (2010). If a worker has minority status, he or she will be subject to greater scrutiny, also increasing the visibility (Kanter, 1977b) of their absences at work. No support was found for predictions derived from gender role theory (Eagly, 1987), which expected all women to show a lower gradient by job level, or derived from a theory of a hegemonic masculinity, (R. W. Connell \& Messerschmidt, 2005; Raewyn W Connell, 1987) assuming that higher level employees in male-dominated occupations will reduce their sickness absence more than other occupational groups. The glass escalator hypothesis highlighted the structurally superior status of men (Blalock, 1967) in female-dominated occupations, but empirically it received no support. For the specific research question at hand, numerical minority status seems to trump role expectations, hegemonic masculinity, and structurally superior status in its predictive power.

Finding a clear gradient for sickness absence by job level is in line with almost all previous studies, although only a few adjusted for other dimensions of stratification were conducted 
in this study (Beemsterboer et al., 2009; Johns, 2010; Pines et al., 1985; Sharp \& Watt, 1995;

Vahtera et al., 2000). The results might at first glance seem to contrast with previous findings that found no support for a disadvantage for the occupational gender minority in terms of sickness absence (Mastekaasa, 2005; Mastekaasa \& Melsom, 2014). However, previous studies focused either on the relationship between sickness absence and job level or between sickness absence and gender (and gender composition), so that we have no direct comparison of the support for the occupational minority hypothesis in the literature.

The results highlight how occupational context interacts with the minority status of groups to modify the vertical stratification within an occupation of a seemingly individual behavior like absenteeism (Paringer, 1983). Interestingly, if the numerical minority is indeed the driving force behind different degrees of stratification, we should be able to predict a similar pattern for ethnic minorities on the (German) labor market without investigating their particular attitudes and behaviors with respect to sickness absence; this suggestion is worthy of examination in future research.

Furthermore, the study highlights the usefulness of the methodological approach of Bayesian evaluation of informative hypotheses. In comparison to standard null hypothesis testing, it makes a virtue out of directly testing the proposed hypotheses and reporting the degree of uncertainty in a way that is much more intuitive to everyday understandings of probability. This can become especially relevant when research results have to be communicated to the public, to non-scientific funding organizations, and to policy makers. Bayesian evaluation of informative hypotheses can be applied in all areas of social sciences and health research which compare groups or use interactions to explain heterogeneous associations. 
The limitations of the study lie, firstly, in the restriction of the analysis to the German labor market. In other labor market settings, the relationship could be different, especially if there is no continuation of wage payments in cases of sickness absence or if gender compositions in occupations are systematically different. Secondly, although they can identify particular groups who might be in need of most support, the analyses do not yet allow the conclusion that promotion or demotion per se are strongest in affecting sickness absence among occupational minorities. This would require the differentiation of selection and causation processes (Hannes Kröger et al., 2015), ideally based on a cohort observed since the beginning of their career. Thirdly, the study does not address the situation of self-employed persons. Although there is no clear equivalent to job level for the self-employed, future research could investigate the reciprocal relationship between the business success of selfemployees and their rates of sickness absence. Fourthly, integrating the workplace level in addition to the occupational level would be an important step forward; characteristics of the employer or supervisor (e.g. gender) can play an important role in structuring the context of the workplace. The last limitation is the potential for interpreting the estimates in this study as causal effects. We argue that the estimates should explicitly not be interpreted as causal effects, but as degrees of stratification. From our theoretical point of view, it might be expected that it is the structural position of jobs with higher authority and autonomy that will induce employees to reduce their sickness absence. However, we also argue that employees will be selected on the same criteria. Both mechanisms are essential components of the stratification. 


\section{$1 \quad 5.1$ Conclusion}

2 We could observe a clear gradient in sickness absence by job level even after taking

3 education, income, and occupational class as major dimensions of stratification into

4 account. The stratification by job level was strongest for occupational minorities, meaning

5 men in female-dominated and women in male-dominated occupations. Both the theoretical

6 approach to the stratification of sickness absence and job level and the method for

7 evaluating the moderating effects of gender and occupational gender composition are applicable to other settings as well. This might apply equally to an investigation of other workplace moderators, cross-country comparisons, or a focus on self-employed individuals. 


\section{References}

(SOEP) Socio-Economic Panel. (2015). data for years 1984-2014, version 31. JOUR. https://doi.org/10.5684/soep.v31.1

Acker, J. (1990). Hierarchies, jobs, bodies: A theory of gendered organizations. Gender \& Society, 4(2), 139-158.

Alexanderson, K., Leijon, M., Åkerlind, I., Rydh, H., \& Bjurulf, P. (1994). Epidemiology of Sickness Absence in a Swedish County in 1985, 1986 and 1987 A Three Year Longitudinal Study with Focus on Gender, Age and Occupation. Scandinavian Journal of Public Health, 22(1), 27-34.

Andersen, H. H., Mühlbacher, A., Nübling, M., Schupp, J., \& Wagner, G. G. (2007). Computation of standard values for physical and mental health scale scores using the SOEP version of SF-12v2. Schmollers Jahrbuch, $127(1), 171-182$.

Baxter, J., \& Wright, E. O. (2000). THE GLASS CEILING HYPOTHESIS A Comparative Study of the United States, Sweden, and Australia. Gender \& Society, 14(2), 275-294.

Beemsterboer, W., Stewart, R., Groothoff, J., \& Nijhuis, F. (2009). A literature review on sick leave determinants (1984-2004). International Journal of Occupational Medicine and Environmental Health, 22(2), 169-179. 
Bekker, M. H. J., Rutte, C. G., \& van Rijswijk, K. (2009). Sickness absence: A gender-focused review. Psychology, Health \& Medicine, 14(4), 405-418.

Blalock, H. M. (1967). Toward a theory of minority-group relations. New York: Wiley.

Blau, P. M. (1977). Inequality and heterogeneity: A primitive theory of social structure (Vol. 7). Free Press New York.

Bratberg, E., Dahl, S.-Å., \& Risa, A. E. (2002). “The Double Burden”: Do Combinations of Career and Family Obligations Increase Sickness Absence among Women? European Sociological Review, 18(2), 233-249.

Formatted: German

Busch, A. (2013). Die berufliche Geschlechtersegregation in Deutschland: Ursachen, Reproduktion, Folgen. Springer.

Clays, E., Kittel, F., Godin, I., Bacquer, D. De, \& Backer, G. De. (2009). Measures of Work-Family Conflict Predict Sickness Absence From Work. Journal of Occupational and Environmental Medicine, 51(8), 879-886.

Cockerham, W. C. (2005). Health Lifestyle Theory and the Convergence of Agency and Structure. Journal of Health and Social Behavior, 46(1), 51-67.

Connell, R. W. (1987). Gender and power: Society, the person and sexual politics. John Wiley \& Sons. 
Connell, R. W., \& Messerschmidt, J. W. (2005). Hegemonic Masculinity Rethinking the concept. Gender \& Society, 19(6), 829-859.

Dean, K. (1989). Self-care components of lifestyles: The importance of gender, attitudes and the social situation. Social Science \& Medicine, 29(2), 137152.

Demerouti, E., Blanc, P. M. Le, Bakker, A. B., Schaufeli, W. B., \& Hox, J. (2009). Present but sick: a three-wave study on job demands, presenteeism and burnout. Career Development International, 14(1), 50-68.

Doress-Worters, P. B. (1994). Adding elder care to women's multiple roles: A critical review of the caregiver stress and multiple roles literatures. Sex Roles, 31(9-10), 597-616.

Eagly, A. H. (1987). Sex differences in social behavior: A social-role interpretation. Psychology Press.

Eagly, A. H., Wood, W., \& Diekman, A. B. (2000). Social role theory of sex differences and similarities: A current appraisal. The Developmental Social Psychology of Gender, 123-174.

Erikson, R., Goldthorpe, H. J., Portocarero, \& Lucienne. (1979). Intergenerational class mobility in three Western European societies:: England, France and Sweden. British Journal of Sociology, 30, 341-415. 
Evans, O., \& Steptoe, A. (2002). The contribution of gender-role orientation, work factors and home stressors to psychological well-being and sickness absence in male- and female-dominated occupational groups. Social Science \& Medicine, 54(4), 481-492.

Ferrie, J. E., Kivimäki, M., Head, J., Shipley, M. J., Vahtera, J., \& Marmot, M. G. (2005). A comparison of self-reported sickness absence with absences recorded in employers' registers: evidence from the Whitehall II study. Occupational and Environmental Medicine, 62(2), 74-79.

Floderus, B., Hagman, M., Aronsson, G., Marklund, S., \& Wikman, A. (2012). Medically certified sickness absence with insurance benefits in women with and without children. The European Journal of Public Health, 22(1), 85-92.

Fried, Y., Melamed, S., \& Ben-David, H. A. (2002). The joint effects of noise, job complexity, and gender on employee sickness absence: An exploratory study across 21 organizations - the CORDIS study. Journal of Occupational and Organizational Psychology, 75(2), 131-144.

Ganzeboom, H. B. G., De Graaf, P. M., \& Treiman, D. J. (1992). A standard international socio-economic index of occupational status. Social Science Research, 21(1), 1-56. 
1 Hoffmeyer-Zlotnik, J. H. P. (2003). "Stellung im Beruf" als Ersatz für eine

2 Berufsklassifikation zur Ermittlung von sozialem Prestige. ZUMA

$3 \quad$ Nachrichten, (53), 114-127.

Hoijtink, H., Klugkist, I., \& Boelen, P. A. (2008). Bayesian evaluation of informative hypotheses. New York: Springer.

Hultin, M. (2003). Some Take the Glass Escalator, Some Hit the Glass Ceiling? Career Consequences of Occupational Sex Segregation. Work and Occupations, 30(1), 30-61.

Jann, B. (2007). Making regression tables simplified. Stata Journal, 7(2), 227.

Jansen, N. W. H., Kant, I. J., van Amelsvoort, L. G. P. M., Kristensen, T. S., Swaen, G. M. H., \& Nijhuis, F. J. N. (2006). Work-family conflict as a risk factor for sickness absence. Occupational and Environmental Medicine, $63(7), 488-94$.

Jarman, J., Blackburn, R. M., \& Racko, G. (2012). The Dimensions of Occupational Gender Segregation in Industrial Countries. Sociology, 46(6), 1003-1019.

Johns, G. (2010). Presenteeism in the workplace: A review and research agenda. Journal of Organizational Behavior, 31(4), 519-542. 
Kanter, R. M. (1977a). Men And Women Of The Corporation, New York: Basic Books.

Kanter, R. M. (1977b). Some Effects of Proportions on Group Life: Skewed Sex Ratios and Responses to Token Women. The American Journal of Sociology, 82(5), 965-990.

Karasek, R. A. (1979). Job Demands, Job Decision Latitude, and Mental Strain: Implications for Job Redesign. Administrative Science Quarterly, 24(2), 285.

Knutsson, A., \& Goine, H. (1998). Occupation and unemployment rates as predictors of long term sickness absence in two Swedish counties. Social Science \& Medicine, 47(1), 25-31.

König, W., Lüttinger, P., \& Müller, W. (1988). A Comparative Analysis of the Development and Structure of Educational Systems. Methodological foundations and the construction of a comparative educational scale (CASMIN-Working Paper No. 10).

Kröger, H. (2016). The contribution of health selection to occupational status inequality in Germany - differences by gender and between the public and private sectors. Public Health, 133, 67-74.

Kröger, H., Pakpahan, E., \& Hoffmann, R. (2015). What causes health inequality? A systematic review on the relative importance of social 
causation and health selection. The European Journal of Public Health, 25(6), 951-960.

Kuoppala, J., Lamminpää, A., Liira, J., \& Vainio, H. (2008). Leadership, Job WellBeing, and Health Effects-A Systematic Review and a Meta-Analysis: Journal of Occupational and Environmental Medicine, 50(8), 904-915.

Kuper, H., \& Marmot, M. (2003). Job strain, job demands, decision latitude, and risk of coronary heart disease within the Whitehall II study. Journal of Epidemiology and Community Health, 57(2), 147-53.

Leijon, M., Hensing, G., \& Alexanderson, K. (2004). Sickness absence due to musculoskeletal diagnoses: association with occupational gender segregation. Scandinavian Journal of Public Health, 32(2), 94-101.

Mackenbach, J. P. (2012). The persistence of health inequalities in modern welfare states: The explanation of a paradox. Social Science \& Medicine, 75(4), 761-769.

Mackenbach, J. P., \& Bakker, M. J. (2003). Tackling socioeconomic inequalities in health: analysis of European experiences. The Lancet, 362(9393), 14091414.

Marmot, M., Feeney, A., Shipley, M., North, F., \& Syme, S. L. (1995). Sickness absence as a measure of health status and functioning: from the UK 
Whitehall II study. Journal of Epidemiology and Community Health, 49(2), 124-30.

Mastekaasa, A. (1996). Unemployment and Health: Selection Effects. Journal of Community \& Applied Social Psychology, 6(3), 189-205.

Mastekaasa, A. (2000). Parenthood, gender and sickness absence. Social Science \& Medicine, 50(12), 1827-1842. f

Mastekaasa, A. (2005). Sickness absence in female- and male-dominated occupations and workplaces. Social Science \& Medicine, 60(10), 22612272.

Mastekaasa, A., \& Melsom, A. M. (2014). Occupational Segregation and Gender Differences in Sickness Absence: Evidence from 17 European Countries. European Sociological Review, 30(5), 582-594.

Nicholson, N., \& Johns, G. (1985). The Absence Culture and Psychological Contract-Who's in Control of Absence? Academy of Management Review, 10(3), 397-407.

North, F., Syme, S. L., Feeney, A., Head, J., Shipley, M. J., \& Marmot, M. G. (1993). Explaining socioeconomic differences in sickness absence: the Whitehall II Study. BMJ, 306(6874). 
Paringer, L. (1983). Women and Absenteeism: Health or Economics? The American Economic Review, 73(2), 123-127.

Peate, I. (2004). Men's attitudes towards health and the implications for nursing care. British Journal of Nursing, 13(9), 540-545. JOUR.

Pierce, J. L. (1996). Gender trials: Emotional lives in contemporary law firms. Univ of California Press.

Pines, A., Skulkeo, K., Pollak, E., Peritz, E., \& Steif, J. (1985). Rates of sickness absenteeism among employees of a modern hospital: the role of demographic and occupational factors. British Journal of Industrial Medicine, 42(5), 326-335. f

Rue, H., Martino, S., \& Chopin, N. (2009). Approximate Bayesian inference for latent Gaussian models by using integrated nested Laplace approximations. Journal of the Royal Statistical Society: Series B (Statistical Methodology), 71(2), 319-392.

Scheil-Adlung, X., \& Sandner, L. (2010). The case for paid sick leave (RPRT). World Health Report (2010) Background Paper,. WHO.

Sharp, C., \& Watt, S. (1995). A study of absence rates in male and female employees working in occupations of equal status. Occupational Medicine, 45(3), 131-136. 
Siegrist, J. (1996). Adverse health effects of high-effort/low-reward conditions. Journal of Occupational Health Psychology, 1(1), 27-41.

Siegrist, J. (2009). Unfair exchange and health: Social bases of stress-related diseases. Social Theory \& Health, 7(4), 305-317.

Simpson, R. (1998). Presenteeism, Power and Organizational Change: Long Hours as a Career Barrier and the Impact on the Working Lives of Women Managers. British Journal of Management, 9, 37-50.

Taylor, C. J. (2010). Occupational Sex Composition and the Gendered Availability of Workplace Support. Gender \& Society, 24(2), 189-212.

Turco, C. J. (2010). Cultural Foundations of Tokenism Evidence from the Leveraged Buyout Industry. American Sociological Review, 75(6), 894-913.

Vahtera, J., Kivimäki, M., Pentti, J., \& Theorell, T. (2000). Effect of change in the psychosocial work environment on sickness absence: a seven year follow up of initially healthy employees. Journal of Epidemiology and Community Health, 54(7), 484-493.

van de Schoot, R., Verhoeven, M., \& Hoijtink, H. (2013). Bayesian evaluation of informative hypotheses in SEM using Mplus: A black bear story. European Journal of Developmental Psychology, 10(1), 81-98. 
Wagner, G. G., Frick, R. J., \& Schupp, J. (2007). The German Socio-Economic Panel Study (SOEP) - Scope, Evolutions and Enhancements. Schmollers Jahrbuch, 127(1), 139-169.

4

Watts, J. H. (2009). Leaders of men: women "managing" in construction. Work, Employment \& Society, 23(3), 512-530.

Williams, C. L. (1992). The Glass Escalator: Hidden Advantages for Men in the "Female" Professions. Social Problems, 39(3), 253-267. 
Tables. 
Table 1 - Names and formal definitions of hypotheses tested in this study

\begin{tabular}{|c|c|}
\hline Name of hypotheses & Formal definition through ordering of coefficients \\
\hline $\boldsymbol{H}_{1 a}$-gender role hypothesis & $\left\{\beta_{m f, r}, \beta_{m m i x, r}, \beta_{m m, r}\right\}>\left\{\beta_{w f, r}, \beta_{w m i x, r}, \beta_{w m, r}\right\}$ \\
\hline $\begin{array}{l}\mathrm{H}_{1 b^{-}}-\text {occupational male culture } \\
\text { of presenteeism hypothesis }\end{array}$ & $\left\{\beta_{m m, r}, \beta_{w m, r}\right\}>\left\{\beta_{m m i x, r}, \beta_{m f, r}, \beta_{w m i x, r}, \beta_{w f, r}\right\}$ \\
\hline $\begin{array}{l}\mathrm{H}_{1 \mathrm{c}}-\text { occupational minority } \\
\text { hypothesis }\end{array}$ & $\left\{\beta_{m f, r}, \beta_{w m, r\}}>\left\{\beta_{m m i x, r}, \beta_{m m, r}, \beta_{w m i x, r}, \beta_{w f, r}\right\}\right.$ \\
\hline $\begin{array}{l}\boldsymbol{H}_{1 d^{-}} \text {glass escalator } \\
\text { hypothesis }\end{array}$ & $\beta_{m f, r}>\beta_{w f, r}$ \\
\hline $\begin{array}{l}\boldsymbol{H}_{2}-\text { Alternative hypothesis (no } \\
\text { ordering) }\end{array}$ & $\left\{\beta_{m m, r}, \beta_{m m i x, r}, \beta_{m f, r}, \beta_{w m, r}, \beta_{w m i x, r}, \beta_{w f, r}\right\}$ \\
\hline
\end{tabular}

Note: The coefficients represent the degree of stratification of sickness absence by job level, adjusted for education, occupational class, and income. $r=\{l, m, h\}$ for lower, middle, and high job level. $m m=$ Men in male-dominated occupations; $m m i x=$ Men in mixed occupations; $m f=$ Men in female-dominated occupations; $w m=$ Women in male-dominated occupations; $w m i x=$ Women in mixed occupations; $w f=$ Women in female-dominated occupations. The study contains three sets of (for each r) six parameters of interest (see table 2). For example, $\beta_{m m i x, l,} \beta_{m m i x, m}$, and $\beta_{m m i x, h}$ represent the stratification of sickness absence by job level (low, middle, and high, respectively) for men in mixed occupations. 
Table 2 - Relative support for the four competing hypotheses - relative differences (negative binomial regression)

\begin{tabular}{|c|c|c|c|c|}
\hline & Expected Probability (\%) & Observed Probability (\%) & Bayes Factor & PM \\
\hline $\boldsymbol{H}_{1 \boldsymbol{a}}-$ gender role hypothesis & 0.0125 & 0.0005 & 0.04 & 0.00 \\
\hline $\boldsymbol{H}_{1 b^{-}}$-occupational male culture of presenteeism hypothesis & 0.0296 & 0 & 0 & 0 \\
\hline $\boldsymbol{H}_{1 c^{-}}$occupational minority hypothesis & 0.0296 & 6.6 & 223 & 0.9 \\
\hline $\boldsymbol{H}_{1 d^{-}}$glass escalator hypothesis & 12.5 & 6.4 & 0.51 & 0.00 \\
\hline $\boldsymbol{H}_{2}$ - Alternative hypothesis (no ordering) & 100 & 0.0005 & 1 & 0.00 \\
\hline
\end{tabular}


Table 3 - Relative support for the four competing hypotheses - absolute differences (linear regression)

\begin{tabular}{|c|c|c|c|c|}
\hline & Expected Probability (\%) & Observed Probability (\%) & Bayes Factor & PMP \\
\hline $\boldsymbol{H}_{1 \boldsymbol{1}}-$ gender role hypothesis & 0.0125 & 0.0015 & 0.12 & 0.0003 \\
\hline $\boldsymbol{H}_{1 b^{-}}$occupational male culture of presenteeism hypothesis & 0.0296 & 0 & 0 & 0 \\
\hline $\boldsymbol{H}_{1 c^{-}}$occupational minority hypothesis & 0.0296 & 11.756 & 397 & 0.9972 \\
\hline $\boldsymbol{H}_{\mathbf{1}} \mathbf{d}^{-}$glass escalator hypothesis & 12.5 & 0.129 & 0.0103 & 0 \\
\hline $\boldsymbol{H}_{2}$ - Alternative hypothesis (no ordering) & 100 & 0.0015 & 1 & 0.0025 \\
\hline
\end{tabular}


Figure 1 - Stratification of sickness absence by job level in relative terms for men and women in three groups of occupational gender segregation

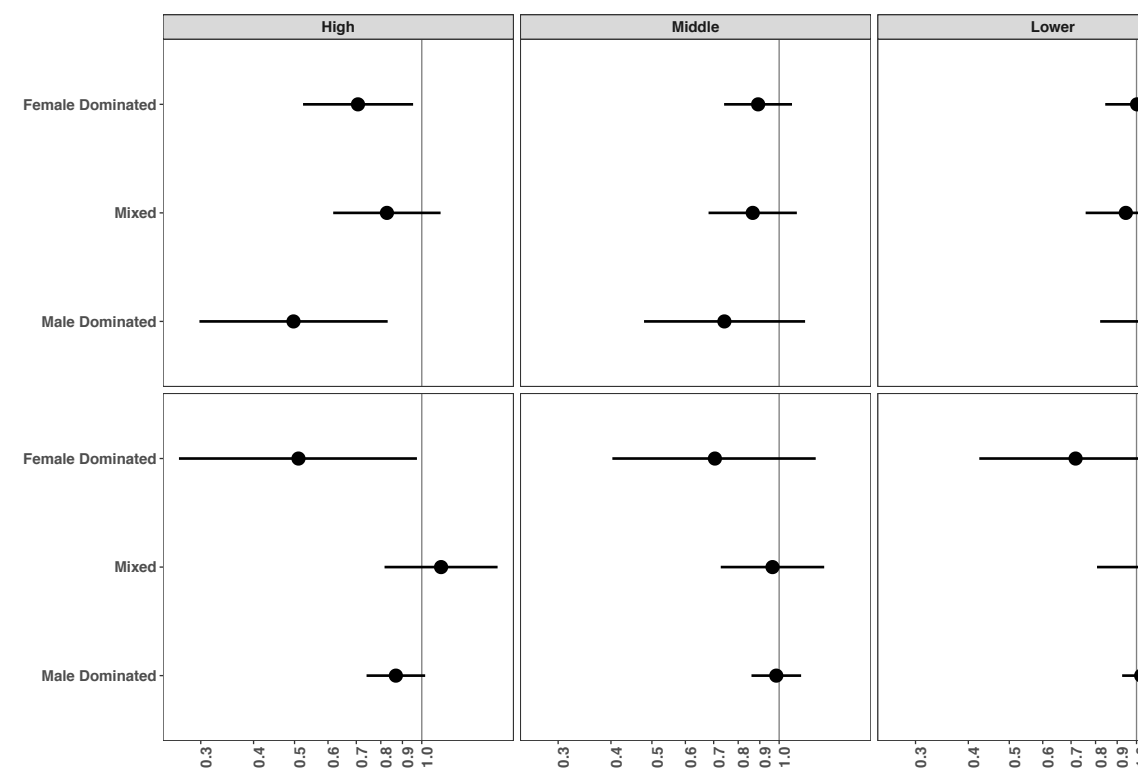

Note: Dots indicate the mean of the posterior distribution of the coefficients as incidence rate ratios (IRR). Lines crossing the dots represent the $95 \%$ credibility interval. 
Figure 2 - Stratification of sickness absence by job level in absolute terms for men and women in three groups of occupational gender segregation

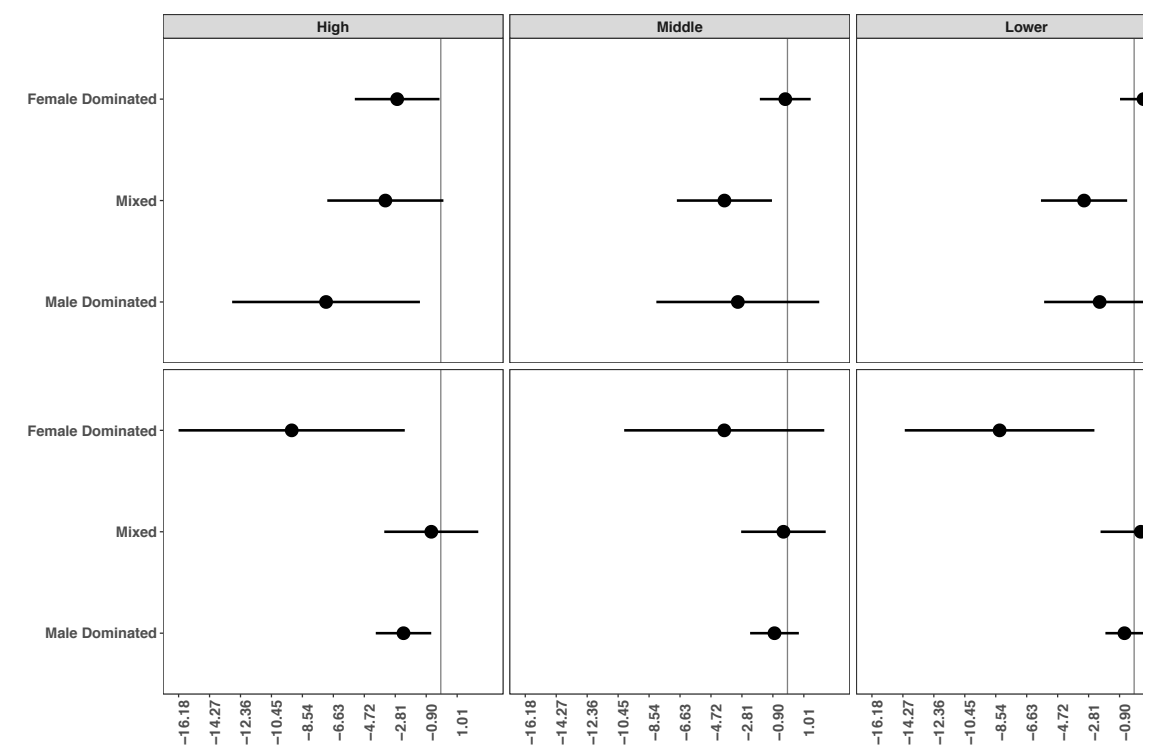

Note: Dots indicate the mean of the posterior distribution of the coefficients as days of sickness absence. Lines crossing the dots represent the $95 \%$ credibility interval. 